\title{
Osteopathie beim Hund - Ruptur des kranialen Kreuzbands
}

\section{Gabriela Klarner}

Ein Kreuzbandriss muss nicht immer operiert werden. Aus Sicht der modernen Faszienforschung ist der Eingriff manchmal sogar kontraproduktiv. Die Osteopathie bietet dafür einen ganzheitlichen Therapieansatz. Anhand des folgenden Fallbeispiels wird die Ursachen-Folge-Kette aufgearbeitet und die Behandlung des kranialen Kreuzbandrisses einer Sheltiehündin vorgestellt.

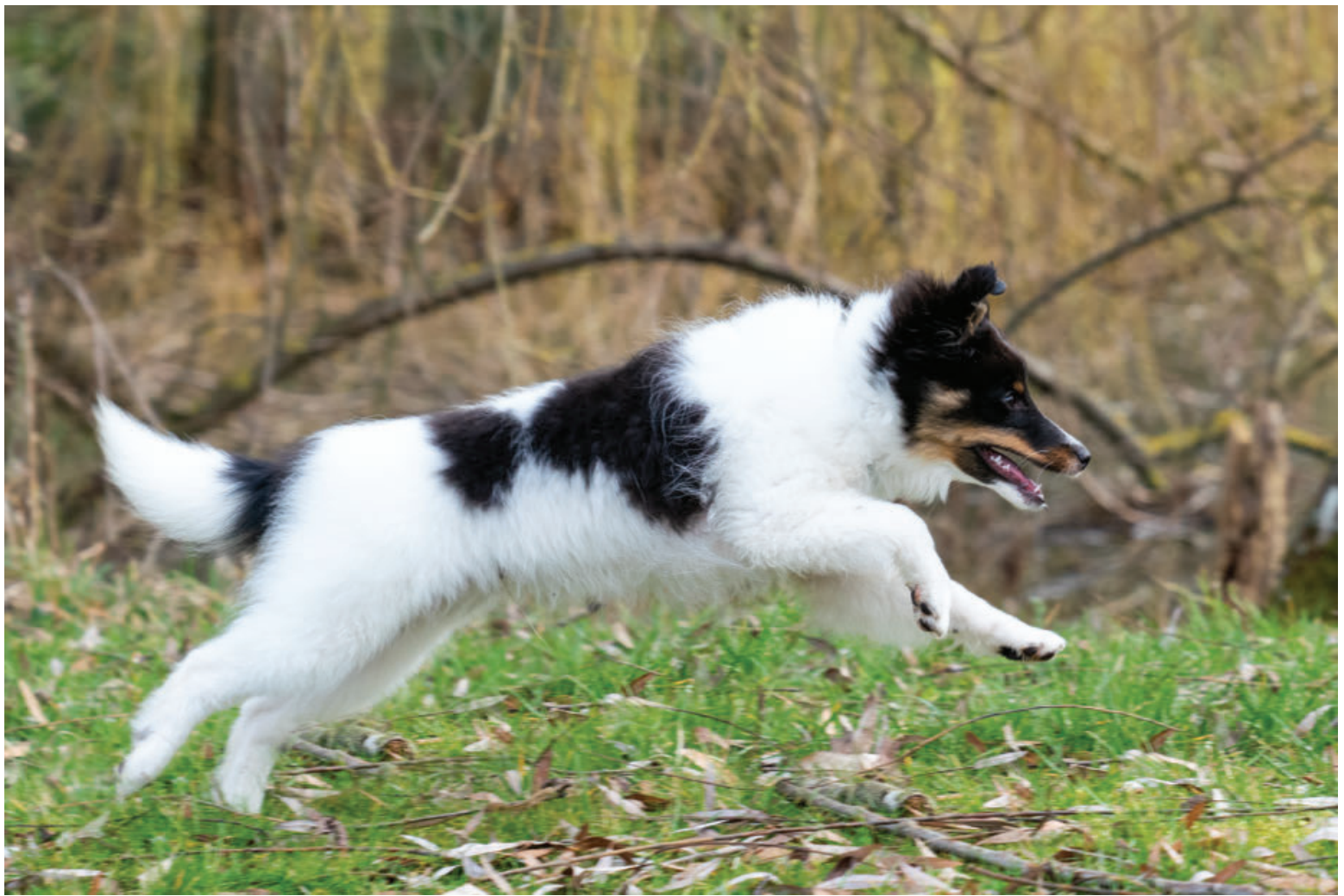

Beim Spielen und Toben zog sich der hier vorgestellte Sheltie eine Ruptur des kranialen Kreuzbands zu, der osteopathisch behandelt wurde. (Symbolbild) Quelle: Kirsten Oborny

Die Ruptur des kranialen Kreuzbands beim Hund ist nicht immer zwingend eine Indikation zur Operation. In sehr vielen Fällen ist es gar nicht das Knie selbst, das die nachhaltigen Probleme bereitet, sondern die Schonhaltungen, die der Patient eingeht, um das geschädigte Knie zu entlasten. Auch eine chirurgische Versorgung des geschädigten Knies löst somit nur bedingt die Lahmheit, mitunter ist eine anschließende physikalische Therapie hilfreich, dennoch zeigen die Hunde immer wiederkehrende intermittierende Lahmheiten auf dem betroffenen Bein. Grund hierfür sind Strukturen, die sowohl in der Chirurgie als auch in der Physiotherapie nicht ausreichend beachtet werden - die Faszien. 
Die Faszien stellen ein bisher völlig unterschätztes Bindeglied zwischen Skelett, Muskulatur, Bandstrukturen, Organen und auch geweblichem Erinnerungsvermögen dar. Das Wissen um den Aufbau, den Verlauf, die topografischen Zusammenhänge dieser Strukturen und die therapeutischen Möglichkeiten an den Faszien, ermöglicht es uns, sogenannte Ursachen-Folge-Ketten zu erstellen und den Patienten ganzheitlich und nachhaltig zu therapieren. Eine chirurgische Intervention ist somit nicht nötig oder bedeutet sogar eine Verzögerung des Heilungsverlaufs aufgrund einer zusätzlichen Gewebeschädigung.

\section{Fallvorstellung}

Vorstellig wurde ein 1-jähriger weiblicher Sheltie, der vor 4 Tagen während des Spielens mit einem anderen Hund plötzlich aufgejault hatte und seitdem links hinten lahm ging. Bei der Erstversorgung beim Tierarzt wurde die Diagnose einer Ruptur des kranialen Kreuzbands im linken Knie gestellt. Ein Röntgenbild ( $\triangleright$ Abb. 1) wurde angefertigt, der Hund wurde auf NSAID gestellt und sollte geschont werden. Eine weitere bildgebende Abklärung wurde aufgrund mangelnder finanzieller Mittel nicht durchgeführt. Ein Termin zur chirurgischen Versorgung wurde vereinbart. Da die Besitzerin des Shelties von der Operation nicht komplett überzeugt war, holte sie eine 2. Meinung ein und wurde bei mir vorstellig.

\section{Der „verfeinerte“ orthopädische Untersuchungsgang}

\section{Gangbildanalyse}

Zunächst wurde ein kompletter orthopädischer Untersuchungsgang durchgeführt. Der Hund wurde im Stehen beurteilt, im langsamen Schritt und im Trab.

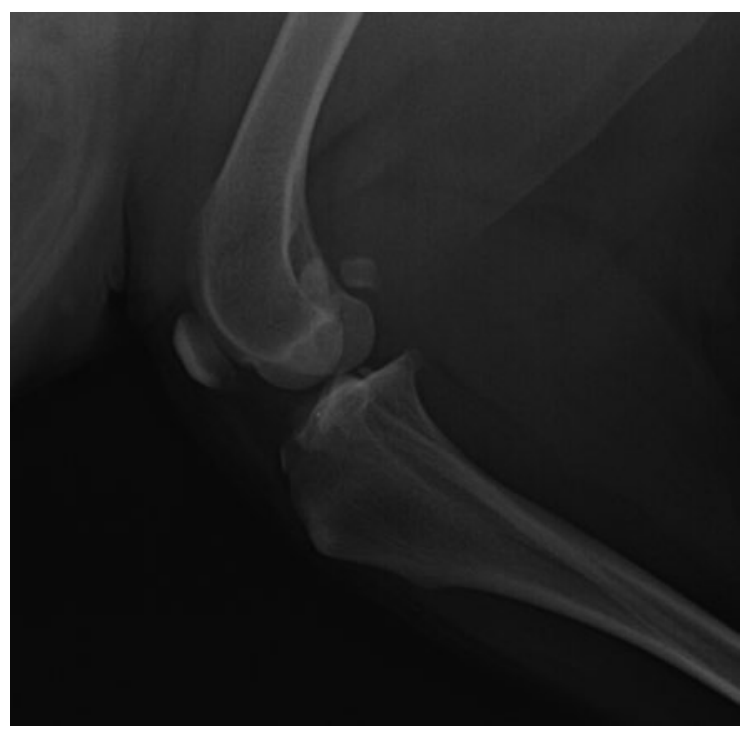

- Abb. 1 Laterale Aufnahme des Kniegelenks. Quelle: Gabriela Klarner
- Im Stehen zeigte sich eine deutliche Entlastung der linken Hinterextremität, indem das Bein gering nach kaudal und das Knie nach lateral ausgestellt wurde. Weiterhin konnte eine leichte Supination im Bereich der Zehen festgestellt werden.

- Im langsamen Schritt wurde eine Stützbeinlahmheit 2. - 3. Grades festgestellt. Alle 3 Phasen der Schrittführung waren verändert: Die Vorführphase war deutlich verkürzt, die Stützphase war kurz und abgehakt und die Stemmphase unvollständig.

- Im langsamen Trab wurde das Bein hochgezogen und nicht belastet.

\section{ENTLASTUNGSHALTUNG}

\section{KNIEGELENKARTHROSE}

Bei fortgeschrittenen Arthrosen im Knie kommt es zu einer deutlich vermehrten Ausstellung der betroffenen Extremität nach kaudal und einer Gewichtsverlagerung nach kranial, was den Schwerpunkt des Hundes nach vorne verlagert und die Unterstützungsfläche vergrößert. Diese Entlastung führt zu einer Schmerzlinderung im betroffenen Knie.

\section{Manuelle Untersuchung der Hinterextremität}

Die manuelle/palpatorische Überprüfung zeigte:

- eine örtlich auf das Knie begrenzte erhöhte Oberflächentemperatur

- eine deutliche Kapselverdickung mit punktuellem medialen Druckschmerz im Bereich der medialen Kollateralbänder

- eine gering vermehrte Gelenkfüllung

- eine deutlich positive Schubladenprobe, bei der sich die Tibia in Relation zum Femur nach kranial verschieben ließ

Die Amplitude dieser Translationsbewegung war groß und bestätigte die Diagnose einer Ruptur des kranialen Kreuzbands.

\section{Beweglichkeit des medialen Meniskus}

In rechter Seitenlage wurde das linke Knie dann von der maximalen Extension langsam in die Flexion geführt und dabei medial am Seitenband durch Auflage von 1 Finger die Beweglichkeit des medialen Meniskus überprüft. Der mediale Meniskus zeigt grundsätzlich eine eingeschränktere Beweglichkeit im Verhältnis zum lateralen Meniskus, da er in Verbindung mit dem medialen Seitenband und der Gelenkkapsel steht und kaudal an der Tibia selbst über das Lig. tibiale caudale menisci mediale fixiert ist. Er folgt somit der Bewegung der medialen Gelenkfläche der Tibia. Diese Bewegung kann man am Beginn der Flexion des Kniegelenks manuell überprüfen. Grundsätzlich bewegen sich die Menisken bei der Flexion nach kaudal. Bei der Hündin zeigte sich eine völlig reibungslose Bewegung. Somit bestand kein Verdacht auf eine Fixation bzw. eine Verletzung des medialen Meniskus. 


\section{Überprüfung in Hyperextension}

Anschließend wurde das Knie in eine Hyperextension geführt (maximale Spannung am Lig. cruciatum craniale) und bei Fixation des Femurs im Bereich der Kondylen versucht, eine Pronation der Tibia durchzuführen. Diese ist nur bei einer Läsion des kranialen Kreuzbands in dieser Position möglich. Hier war auch diese Probe positiv.

\section{Untersuchung der Hüfte}

Die Hüfte war in der Rotation bei exakter Handanlage am Oberschenkel (und schmerzbedingt nicht am Knie!) nicht eingeschränkt und auch nicht schmerzhaft, die einzelnen Ebenen wurden nochmals überprüft. Flexion und Extension (Sagittalebene), Abduktion und Adduktion (Transversalebene) und Pro- und Supination waren ohne Befund.

\section{Der osteopathische Untersuchungsgang}

Anschließend wurde ein osteopathischer Untersuchungsgang mit besonderem Augenmerk auf die faszialen Strukturen durchgeführt.

\section{Anatomie und Topografie}

Die dorsale Faszienkette des Rumpfes hat ihren Ursprung in Form der Fascia cervicalis superficialis am Okziput, an der Mandibula und am Processus zygomaticus. Sie setzt sich in Form der Fascia thoracolumbalis, der Fascia glutea, Fascia coccygea, der Fascia lata bis in die Fascia cruris fort. Diese zieht bis an den Tarsus. Ihre Aufgabe ist die Extension der gesamten Wirbelsäule.

Die ventrale Faszienkette des Rumpfes hat ihren Ursprung am Os hyoideum. Sie umgibt die Halsbeuger in Form der Fascia cervicalis profunda, setzt sich fort in der Fascia abdominalis und Fascia transversa abdominis zum Tendo praepubicus und weiter in die Fascia iliaca. Ihre Aufgabe ist die Flexion der gesamten Wirbelsäule.

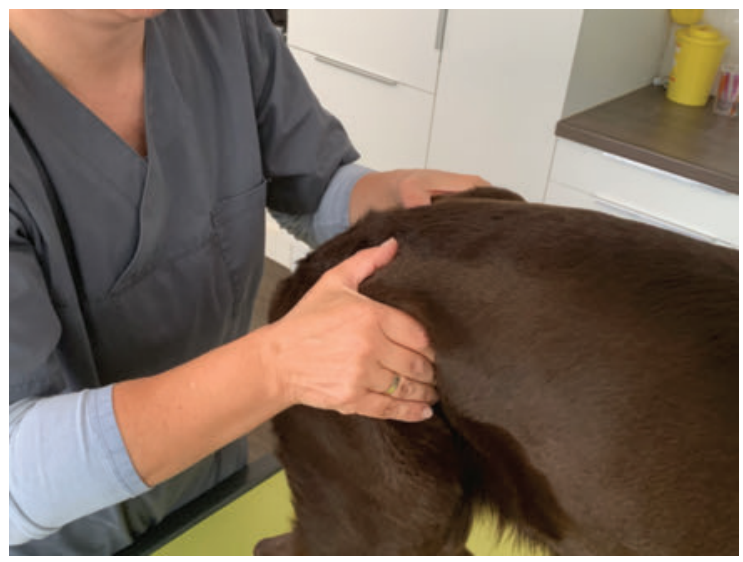

- Abb. 2 Zug an der dorsalen Faszienkette. Die Fotos wurden am Labrador angefertigt, da durch die Fellqualität beim Sheltie die Handanlagen nicht darstellbar wären. Quelle: Gabriela Klarner
Diese Faszien sind aber auch für die Schrittführung der Hintergliedmaße von maßgebender Bedeutung. So sind beim Vorführen der Hinterextremität die Fascia lata und die Fascia iliaca unter Spannung, die Fascia glutea und cruris sind hingegen relaxiert. In der Stemmphase sind die Fascia glutea und Fascia femoralis unter Spannung und die Fascia lata und iliaca relaxiert.

\section{Untersuchung}

Um Spannungen im Verlauf der dorsalen Kette festzustellen, wird mittels Handanlage an den Oberschenkel beidseits kranial im Bereich der Kniefalte der Verlauf der Faszien überprüft. Dabei werden Verdichtungen, Falten und Spannungen festgestellt ( $\bullet$ Abb. 2). Alternativ kann man durch Handanlage dorsal im Bereich des Sakrums diese Veränderungen feststellen, indem man sich auf die Faszienebene begibt und deren Verlauf folgt ( $\triangleright$ Abb. $\mathbf{3}$ ).

Die ventrale Kette wird mittels Handanlage am Tendo praepubicus in gleicher Weise auf Veränderungen überprüft ( $\triangleright$ Abb.4). Festzuhalten ist hierbei, dass die ventrale Faszienkette des Rumpfes über die Fascia iliaca eine Verbindung zum M. obliquus externus hat und dieser wiederum über eine Aponeurose mit der Fascia femoralis medialis verbunden ist. Diese zieht mit ihren tiefen intramuskulären Septen bis an die Medialseite des Femurs und inseriert dort.

Aus der Schonhaltung des Knies ergibt sich grundsätzlich eine vermehrte Spannung (Auswärtsrotation). Diese Spannung setzt sich direkt auf den Wirbelkörper des 4. Lendenwirbels fort und bringt diesen in eine Extensionsstellung mit einer Rotation nach links ( $\mathbf{A b b}$.5).

\section{Überprüfung mittels struktureller Osteopathie}

Bei der Untersuchung ermittelte Befunde werden anschließend auch strukturell noch überprüft. In der strukturellen

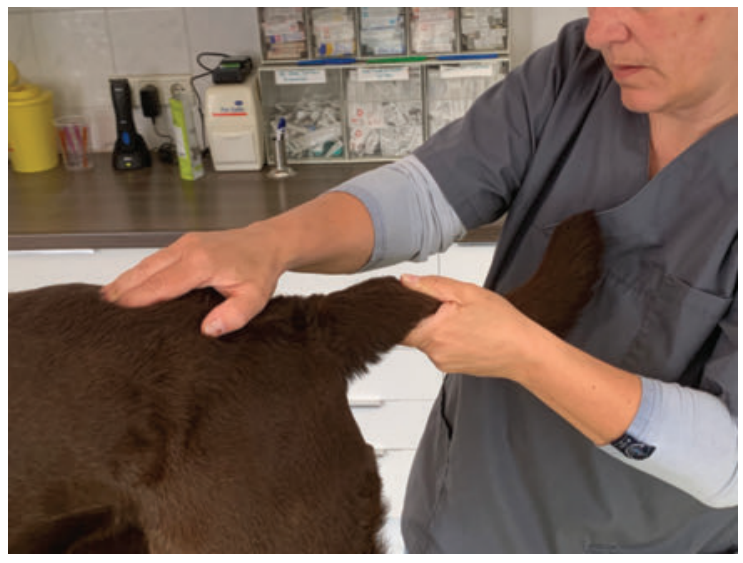

- Abb. 3 Überprüfung der dorsalen Faszienkette mittels Handanlage am Sakrum und sanftem Zug am Schweif, um die Kette zu spannen. Quelle: Gabriela Klarner 


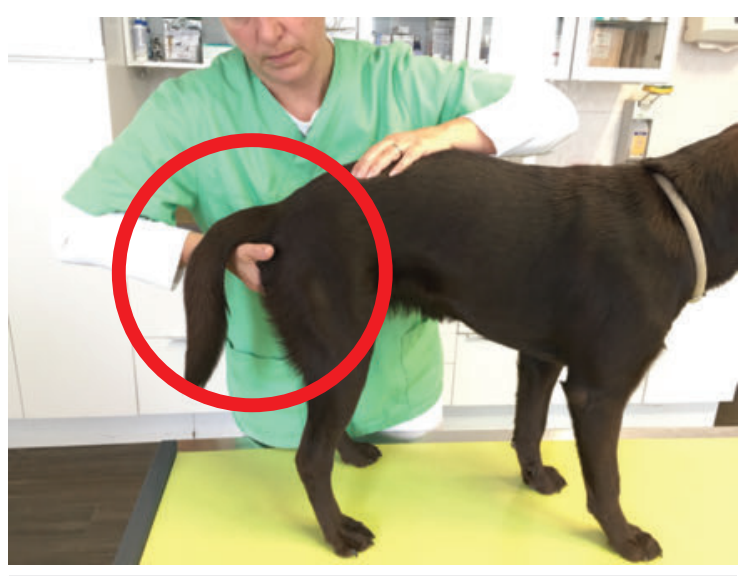

- Abb.4 Überprüfung der ventralen Kette durch Handanlage am Tendo praepubicus. Quelle: Gabriela Klarner

Osteopathie arbeitet man zunächst im Bereich der Wirbelsäule und überprüft die Stellung der Wirbel im Einzelnen.

\section{DEFINITION}

In der Terminologie der Osteopathie kommt es bei einer Extensionsstellung eines Wirbels zu einer Annäherung der Processus spinosi zum vorangehenden Wirbel (n-1). Die Rotation wird nach der Unterseite des Wirbelkörpers benannt (Rotation nach links = Verlagerung des Processus spinosus nach rechts) und bei einem Sidebending kommt es zu einer Annäherung der Processus transversi der Wirbel $\mathrm{n}$ und n-1. Dabei erhöht sich die Spannung der Ligg. flava auf der kontralateralen Seite und es kommt zu einer Verdichtung dieser auf der konkaven Seite.

\section{Befunde der Hündin}

Der 4. Lendenwirbel wurde in der oben beschriebenen Extensionsstellung vorgefunden, Rotation und Sidebending nach links. Durch die Gewichtsverlagerung nach kranial kommt es zu einer Überlastungsblockade der diagonalen Vorderextremität. Die rechte Schulter war in ihrer Abduktion und Extension eingeschränkt, die Vorführphase verkürzt und die umgebende Muskulatur angespannt.

Die Überprüfung des thorakoskapulären Gleitens zeigte eine deutliche Einschränkung der Amplitude. Dabei wird der Hund in die linke Seitenlage gebracht und beruhigt, sodass die aufregungsbedingte Muskelspannung nachlässt und anschließend mit einer Hand am Buggelenk und mit der anderen Hand an der dorsalen Kontur der Skapula gefasst. Nun wird die Beweglichkeit der Skapula in Relation zum Thorax in kranialer und kaudaler Richtung überprüft [1].

Der M. omotransversarius und der M. brachiocephalicus stehen bei der Sheltiehündin rechts unter deutlich

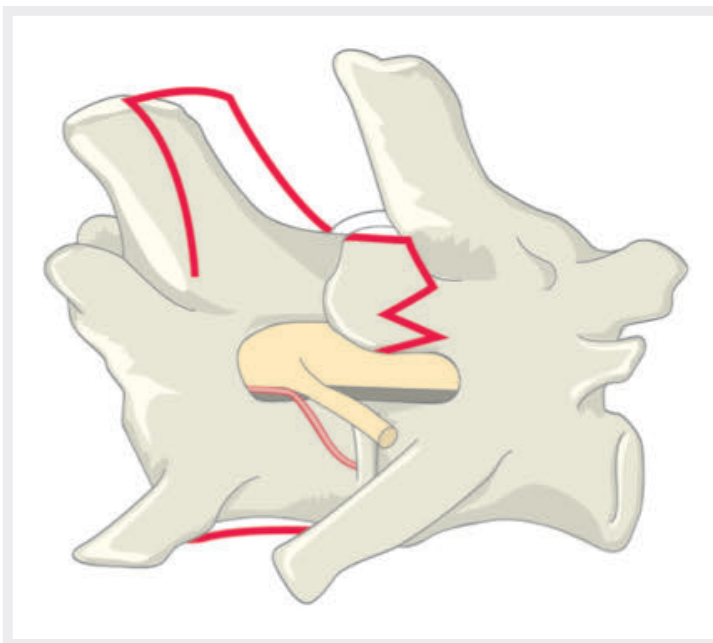

- Abb. 5 Bei einer Extensionsstellung des Wirbels kommt es zur Annäherung der Processus spinosi zum vorangehenden Wirbel und zu einer Überlappung der Gelenkfacetten. Bei einer zusätzlichen Rotation nach links wird der linke Processus transversus nach dorsal verschoben. Quelle: Thieme Gruppe nach einer Vorlage von Gabriela Klarner

vermehrter Kontraktur. Der M. omotransversarius zieht an seinem Ansatz auch in die Fascia brachii. Diese Faszie umgibt die Muskeln des Oberarms und teilt sich in ein kraniales Kompartiment, welches die Flexoren umgibt und in ein kaudales Kompartiment, welches die Extensoren umgibt. Die Kontraktur dieser Muskeln schränkt die Vorführphase der rechten Vorderextremität ein. Die Spannung führt zusätzlich über den Ursprung des M. omotransversarius an den Querfortsätzen des Atlas und Axis rechts zu einer leichten Rotation nach links.

Folgende osteopathische Befunde wurden abhängig von dem ursprünglichen Knieproblem erhoben:

- fasziale Faltenbildung in der dorsalen Kette im Bereich des L4

- L4 in Extension, Rotation und Sidebending nach links

- Spannungserhöhung an der Fascia femoralis medialis links und weiter über die Aponeurose in den M. obliquus externus links

- Blockade des Schultergelenks rechts

- Kontraktur des M. omotransversarius und M. brachiocephalicus rechts

- Spannung in der Fascia brachii rechts

- C1 in Rotation nach links

- Bewegungseinschränkung L1/2 mit Vektorspannung ins Diaphragma

- Blockade der Articulatio costovertebralis der letzten beiden Rippenköpfchen links

- Einschränkung des primären Atemmechanismus (s. u.) im Kniegelenk 


\section{DIAPHRAGMA - BETEILIGUNG AN \\ OSTEOPATHISCHEN LÄSIONEN}

Die dorsale Aufhängung des Diaphragmas beim Hund erfolgt überkreuz am L1 und L2. Eine Spannung im Zwerchfell kann zu einer leichten Bewegungseinschränkung der beiden Wirbel führen, die manuell ertastet werden kann. Zudem verdichtet sich der M. iliopsoas in diesem Bereich. Die Spannung in den beiden oberen Quadranten des Diaphragmas steht in Verbindung mit der veränderten Atmung bei Schmerzen.

\section{Therapie und Behandlungsverlauf}

Gegen eine kurzzeitige Behandlung mit NSAID ist kein Einwand zu erheben. Sie beschleunigt den akuten Entzündungs- und Schmerzprozess in der Heilung. Immer vorausgesetzt, der Hund verträgt die Medikamente vom Magen-Darm-Trakt her.

\section{Osteopathische Therapie}

Die Faszienfalten werden durch Entrollen der Faszienkette beidseits über die Hinterhand geglättet. Dabei wird der Hund stehend am eigenen Körper fixiert und jeweils an der kontralateralen Extremität gearbeitet. Dazu wird die Extremität in die Streckung nach kaudal gebracht, die Faszienebene fokussiert und dem Verlauf der Faszie gefolgt. Zur besseren Fokussierung kann man mit der kranialen Hand im Bereich der Falte einen Orientierungspunkt mit den Fingern setzen, indem man mit der Hand unter dem Bauch des Hundes durchgreift. Folgt man den Bewegungen dieser Faszie bis ins Release, ist die Faszienfalte verschwunden ( $\bullet$ Abb.6).

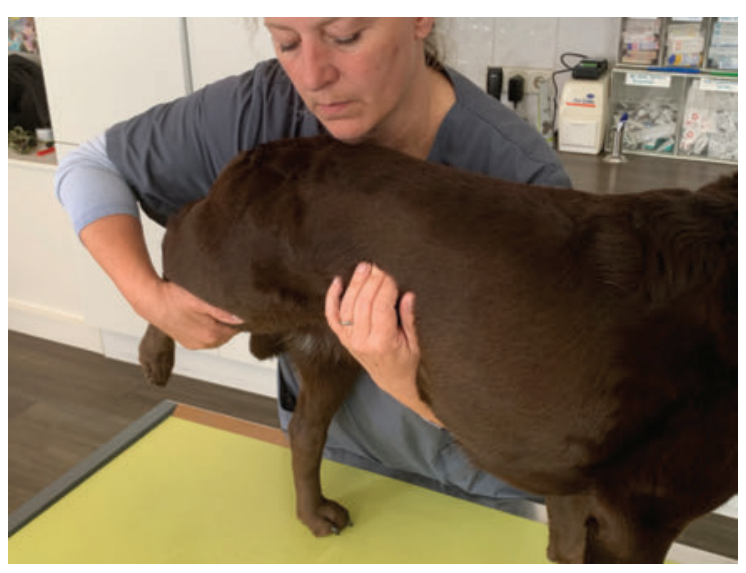

- Abb. 6 Entrollen der Faszien über die Hinterextremität mit punktueller Fixation des Segments mit der Falte. Quelle: Gabriela Klarner

\section{DEFINITION}

Unter einem Release versteht man das Ende der Entrollung von Fasziengewebe. Diese fühlt sich an wie der Übergang von einer ungeordneten pulsierenden Bewegung in eine rhythmische sanfte Kontraktion. Das Release signalisiert dem Therapeuten eine erfolgreiche Lösung vorhandener Fehlspannung im Gewebe. Die Struktur wird weich und entspannt.

In derselben Handanlage kann man die Spannung auf der Fascia femoralis medialis mittels Resilience-Technik wegschmelzen. Die Lösung jeder einzelnen Läsion wird der Patient mit Schlecken oder Gähnen bestätigen, was zeitgleich auch für den Therapeuten eine zusätzliche Bestätigung für den Erfolg seiner Manipulation darstellt.

Der L4 hat sich schon allein durch diese Manipulation ein wenig aus der Extensionsstellung erhoben. Zur kompletten Reposition wird ein punktueller Druck auf den Processus spinosus des $\mathbf{L} 4$ in dorsoventraler Richtung durchgeführt. Dabei wird die Läsion kurzfristig verstärkt und die Blockade indirekt gelöst (im Sinne der Lösung einer alten Handbremse, die man, um sie zu lösen, nochmal stärker anziehen muss). Auch dazu fixiert man den Hund so, dass er dieser Bewegung nicht ausweichen kann, indem man ihn mit einer Hand unter dem Bauch an den Hinterextremitäten festhält. Der Druck ist vergleichbar damit, dass man einen On-/Off-Schalter betätigt ( $\vee$ Abb. 7).

Anschließend wird mittels Resilience-Technik die leichte Bewegungseinschränkung der Wirbel L1 und L2 gelöst. Die Verdichtungen im Diaphragma und im M. iliopsoas werden mit derselben Technik bis zum Verschwinden eingeschmolzen.

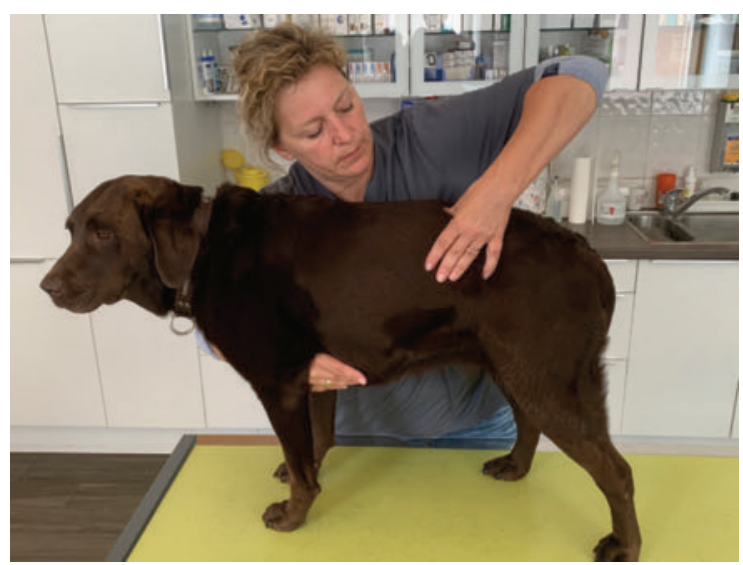

- Abb. 7 Strukturelle Lösung des L4 aus der Extensionsstellung. Quelle: Gabriela Klarner 


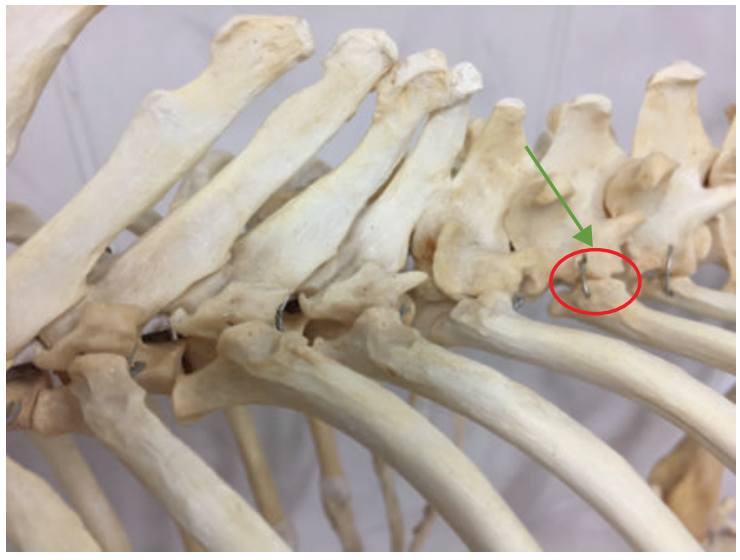

- Abb. 8 Gelenke der Rippenköpfchen (Kreis); Pfeil: Druckrichtung bei der Reposition. Quelle: Gabriela Klarner

Die blockierten Rippenköpfchengelenke links kaudal werden punktuell durch kurzen, schnellen Druck von dorsal nach ventrolateral reponiert ( $\bullet$ Abb. 8). Um ein Nachgeben bei der Reposition zu verhindern, wird mit der anderen Hand von ventral an der Spitze des Sternums gegengehalten. Meist wird diese Reposition von den Hunden nicht sehr gerne toleriert, aber durch die Schnelligkeit der Aktion (Thrust) werden sie so überrascht, dass sie mit einer Abwehrbewegung immer zu spät sind.

\section{Merke}

Bitten Sie den Besitzer für diese Manipulation trotzdem kurzzeitig den Kopf zu fixieren oder setzen Sie dem Hund einen Maulkorb auf.

Die muskuläre Kontraktur rechts seitlich am Hals wird durch sanfte Dehnung gelöst, dazu wird der Hund wieder am eigenen Körper stabilisiert und kontralateral gearbeitet. Die kraniale Hand greift von oben mit der Längsseite des Zeigefingers an die kaudale Kante der Mandibula, die kaudale Hand greift breit im Bereich des Schultergelenks rechts. Die beiden Hände werden langsam und sanft voneinander wegbewegt, die dazwischenliegenden Strukturen vorsichtig gedehnt und dabei wird der Kopf des Hundes langsam nach links gebogen ( Abb.9). Oft spürt man in dieser Handanlage wieder eine Unwinding-Bewegung der seitlichen Halsfaszie, der man unbedingt nachgeben sollte. Mit dem Release dieser Bewegung spürt man auch die deutliche Entspannung der muskulären und faszialen Strukturen. Der Patient quittiert die Lösung der Blockade wieder mit deutlichem Schlecken oder Gähnen.

Diese Position halten, mit der kaudal liegenden Hand an den 2. oder 3. Halswirbel greifen und diesen am Processus transversus fixieren. Die kranial liegende Hand greift nun von dorsal auf den Fang des Hundes und durch eine sichere Drehbewegung nach rechts in Flexion der Halswirbelsäule wird die Rotationsblockade von C1 direkt mittels Thrust eröffnet $(\bullet \mathbf{A b b} \mathbf{b} \mathbf{1 0})$.

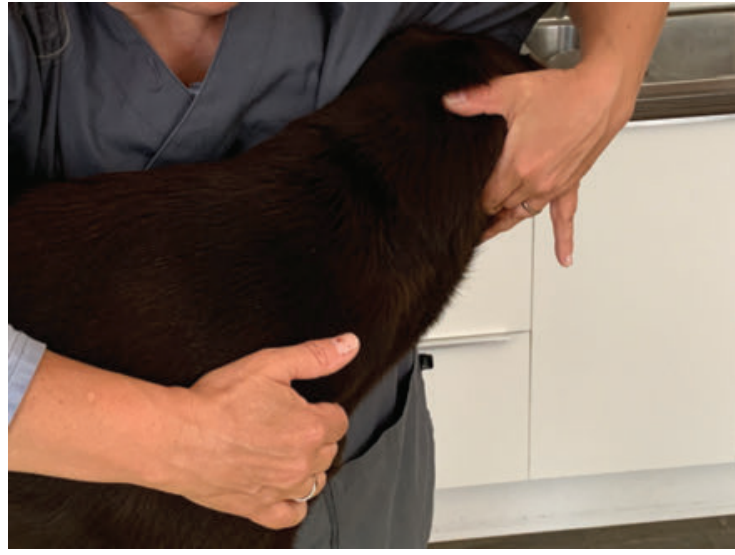

- Abb.9 Dehnung der seitlichen Halsmuskulatur und der seitlichen Halsfaszie. Quelle: Gabriela Klarner

Der Hund wird weiterhin am eigenen Körper fixiert und mit der kranialen Hand an das Ellbogengelenk der rechten Vorderextremität von kraniolateral gegriffen. In Streckstellung mit Fokussierung auf die Faszie wird die Fascia brachii über die Vorderextremität entrollt. Anschließend wird das Bein langsam abgesetzt, der Therapeut wechselt die Position und stellt sich vor den Hund. Dabei greift die rechte Hand des Therapeuten distal an die Extremität des Hundes in Höhe des Unterarms, die linke Hand des Therapeuten fixiert die Skapula von lateral in Höhe des Akromions. Die Extremität des Hundes zeigt auf die Mittelachse des Therapeuten und in Streckstellung des Schultergelenks wird nach Spannungsaufbau mittels Thrust nach lateral die Gelenkblockade gelöst. Alle Korrekturen werden im Anschluss nochmals auf ihre Vollständigkeit überprüft.

Als Abschluss wird mittels PAM-Technik (PAM: primärer Atemmechanismus) direkt am Knie gearbeitet und die Amplitude des primären Atemmechanismus vergrößert, um die Regeneration zu forcieren. Dabei erfühlt man den primären Atemmechanismus des Gelenks und hält jeweils an den Endpunkten der Amplitude die Bewegung minimal zurück. Zug um Zug vergrößert sich dabei die Amplitude. Der primäre Atemmechanismus kann nach Sutherland in jedem Organ erfühlt werden und stellt eine Form einer harmonischen, rhythmischen Schwingung dar, vergleichbar mit dem Ein- und Ausatmen (steht aber nicht mit der Atmung an sich in Verbindung). Diese Bewegung nutzt zur Fortbewegung vorrangig fasziale Strukturen.

\section{Weiterer Behandlungsverlauf}

Die Sheltiehündin wurde 4-mal jeweils im Abstand von 14 Tagen behandelt. Die Besitzerin wurde angehalten, den Hund nur an der Leine zu führen und keine Treppen steigen zu lassen. Die NSAID wurden nach 5 Tagen abgesetzt, da der Hund mit kolikartigen Magenschmerzen reagierte.

Das Knie zeigte schon bei der 2. Behandlung keine Entzündungssymptome mehr, die Kapselverdickung war 


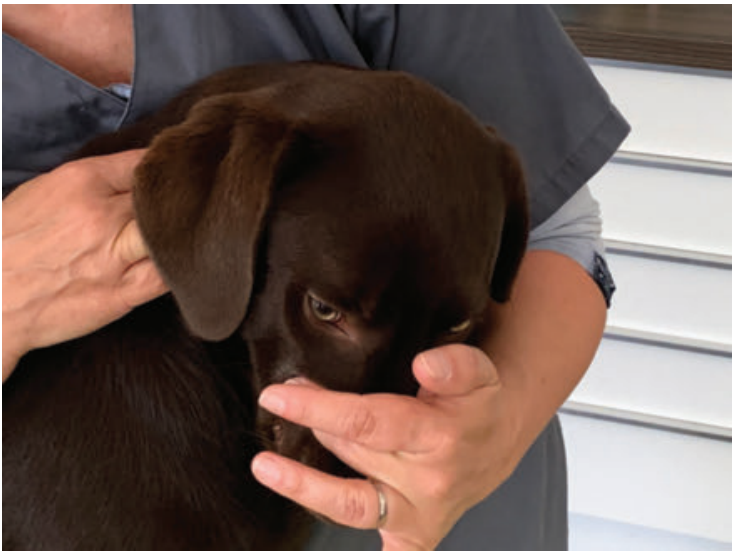

- Abb.10 Rotation des Kopfes nach rechts und direkte Eröffnung der Rotationsblockade des C1. Quelle: Gabriela Klarner

verschwunden und auch die vermehrte Gelenkfüllung war nicht mehr vorhanden. Die Schubladenprobe war nach wie vor positiv, allerdings wurde die Amplitude deutlich geringer und auch die Schmerzhaftigkeit war fast vollständig verschwunden. Bei der 2. und 3. Behandlung war jedes Mal der L4 wieder in der Extension, die Ausgleichslahmheit rechts vorne blieb aber verschwunden. Die Faszienfalten waren in unterschiedlicher Stärke bei jeder Behandlung vorhanden, mitunter auch an mehreren Stellen. Die Hündin war nach der 4. Behandlung komplett symptomfrei, die Schubladenprobe war negativ.

Mit der Besitzerin wurde eine langsame Steigerung der Bewegung vereinbart, zunächst war kontinuierliches Traben angesagt und gezieltes Bewegungstraining in Form von Übungen, die die Oberschenkelmuskulatur stärkten. Anschließend wurde Training am Balancekissen verordnet. Eine Unterwassertherapie war wegen der Wasserscheue des Hundes nicht möglich.

Die Hündin wurde 6 Monate später wieder vorstellig. Sie hatte sich beim Spielen das kraniale Kreuzband des rechten Knies rupturiert. Auch hier konnte ein chirurgisches Vorgehen vermieden werden und die Hündin wurde erfolgreich konservativ/osteopathisch therapiert. Zu beachten ist in diesem speziellen Fall, dass das Gewicht des Patienten < $10 \mathrm{~kg}$ liegt, was eine deutliche Erleichterung der Therapie darstellt. Der Patientenbesitzer war zudem kooperativ und sehr bemüht. Die Sheltiehündin kommt seitdem alle 4 Monate regelmäßig zur Therapie, der Vorfall liegt 4 Jahre zurück. Sie ist aber seither komplett lahmheitsfrei und aktiv als Begleithund im Einsatz.

\section{Fazit}

Nicht jeder Kreuzbandriss ist operativ zu kurieren, manchmal ist ein chirurgisches Einschreiten vielleicht sogar kontraproduktiv. Bei den gängigen Operationsmethoden, z. B. Cleaning up, lateraler Haltebandersatz, TPLO (Tibial Plateau
Leveling Osteotomy), kommt es zu keiner Wiederherstellung der Bandstrukturen. Bei der TPLO kommt es sogar zu einem zusätzlichen kontrollierten Knochentrauma und einer Veränderung der Winkelung im Knie und in weiterer Folge auch an der gesamten Extremität. Bei großen Hunden und bei Hunden, die schwer in ihrer Bewegung zu kontrollieren sind, ist für jede dieser Operationsmethoden eine vorsichtige Prognose zu stellen.

\section{TAKE HOME}

Aus Sicht der modernen Faszienforschung sollte generell jeder Eingriff neu überdacht werden. Jedes noch so kleine fasziale Trauma wird eine Kette von geweblichen Ereignissen nach sich ziehen. Faszien bilden ein bindegewebiges Netzwerk im Körper, das sowohl fibrilläre als auch histologische Kontinuität aufweist. Sie haben ihre Aufgaben im Kreislauf, im Nervensystem, im Immunsystem, in der Biomechanik und auch als Transportmedium. Ohne Faszien wären wir nur ein Haufen zusammengewürfelter Zellen. Sie sorgen für die mechanische und funktionelle Einheit des Körpers. Mit diesem Bewusstsein sollten wir unsere Therapieansätze neu überdenken und unser chirurgisches Einschreiten exakter abwiegen.

\section{Autor}

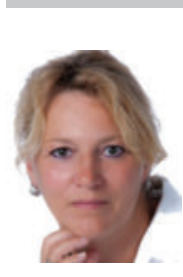

\section{Gabriela Klarner}

Dipl. Tierärztin; 2017 Graduierung EVSO-C; Komiteemitglied der EVSO; 2019 Gründerin der AVSOP - Austrian Veterinary School of Osteopathy (www.avsop.at); seit 2007 in eigener Kleintierpraxis in Wien; www.tierordination-elderschplatz.at; praxis@ toe.cc

Literatur

[1] Hohmann M. Bewegungsapparat Hund. 2. Aufl. Stuttgart: Sontag; 2018

[2] Guimberteau JC. Faszien - Architektur des menschlichen Fasziengewebes. Berlin: KVM - Der Medizinverlag; 2016

[3] Hames JM. Faszien. Lehrmaterial aus der Grundausbildung Osteopathie für Tierärzte: 2016

[4] Pallandre JP. Veterinary manual medicine, macrodynamic techniques, the hindlimb. Lehrmaterial aus der Grundausbildung Osteopathie für Tierärzte

[5] Hartmann C, Hrsg. Das große Sutherland-Kompendium. Pähl: Jolandos; 2013

\section{Bibliografie}

DOI https://doi.org/10.1055/a-1046-2241

Hands on 2020; 2 : 32-38

(c) Georg Thieme Verlag KG Stuttgart · New York ISSN 2628-6033 